International Journal of Pure and Applied Mathematics

Volume 108 No. 1 2016, 159-168

ISSN: 1311-8080 (printed version); ISSN: 1314-3395 (on-line version)

url: http://www.ijpam.eu

doi: 10.12732/ijpam.v108i1.14

ijpam.eu

\title{
STOCHASTIC BLACK-SCHOLES EQUATION WITH TIME-FRACTIONAL DERIVATIVE ON THE HALF-LINE
}

\author{
J. Sanchez-Ortiz ${ }^{1}$, M.P. Arciga-Alejandre ${ }^{2} \S$, \\ F.J. Ariza-Hernandez ${ }^{3}$, J.C. Hernandez-Pastrana ${ }^{4}$ \\ 1,2,3,4 Faculty of Mathematics \\ University of Guerrero \\ Chilpancingo, 39087, Guerrero, MEXICO
}

\begin{abstract}
We investigate the pricing of options using a modified Black-Scholes equation with a time-fractional derivative and additive white noise on the half-line. We construct the Green function for the initial-boundary value problem adapting the main ideas of the Fokas method and we prove existence and uniqueness of solutions.
\end{abstract}

AMS Subject Classification: $35 \mathrm{C} 15,35 \mathrm{~A} 22,60 \mathrm{G} 22$

Key Words: black-scholes, Fokas method, Caputo fractional derivative, Mittag-Leffler function

\section{Introduction}

Black and Scholes [2] proposed a theoretical model to determine the valuation of an European or American option of type call or put, on a non-dividend paying stock. The Black-Scholes equation it is given by

$$
\frac{\partial V}{\partial \tau}+\frac{1}{2} \sigma^{2} S^{2} \frac{\partial^{2} V}{\partial S^{2}}+r S \frac{\partial V}{\partial S}-r V=0,(S, \tau) \in \mathbb{R}^{+} \times[0, T],
$$

where $V(S, \tau)$ is the value of the option, $S$ is the price of the underlying asset, $\tau$ is the time, $T$ is the expiration date, $\sigma$ is the volatility of the underlying asset and $r$ is the risk-free interest rate. A great number of works has been devoted to study this model through different points of view, (see for instance [1], [6], [13]). On the other hand, some authors have generalized this model,

Received: March 8, 2016

Published: June 6, 2016

${ }^{\S}$ Correspondence author (c) 2016 Academic Publications, Ltd.

url: www.acadpubl.eu 
replacing the geometric Brownian motion by fractional Brownian motion, see [8], [9], [14]. Other generalization is obtained by changing the partial derivative in the time variable by a fractional derivative, see [3], [7], [11]. In comparison with standard derivatives of integer order, the fractional order derivatives are characterized by their memory, i.e., the rate of change of a function near a point is affected by the history in the time domain of definition rather than just near the point itself.

Lets notice that the equation (1) is equivalent, via the change of variables: $V(S, \tau)=K q(x, t), S=K e^{x}, \tau=T-t$, with $K>0$ a constant, to the equation

$$
q_{t}=a q_{x x}+(b-a) q_{x}-b q,
$$

where $a=\frac{1}{2} \sigma^{2}, b=r$. In this work, we consider an initial-boundary value problem for a stochastic evolution equation of Black-Scholes type with timefractional derivative and white noise on the half-line,

$$
\left\{\begin{array}{l}
\mathcal{D}_{t}^{\alpha} q=a q_{x x}+(b-a) q_{x}-b q+\mathcal{N} q+\dot{B}, \quad x>0, t \in[0, T], \\
q(x, 0)=q_{0}(x) \\
q(0, t)=g_{0}(t)
\end{array}\right.
$$

where $\mathcal{D}_{t}^{\alpha}$ is the Caputo fractional derivative with $0<\alpha<1 ; \mathcal{N}$ is a lipschitzian operator and $\dot{B}(x, t)$ is the white noise on $\mathbb{R}^{+} \times[0, T]$.

This paper is organized as follows: First, we solve the linear problem associated with (2), adapting the main ideas of the Fokas method [4] for fractional differential equations, we construct the Green's function. Then, by Duhamel's principle we propose an integral representation for the solution of (2) and by virtue of Gronwall's lemma we are able to prove existence and uniqueness.

\section{Preliminaries}

In this paper, the $\dot{B}(x, t)$ is the white noise on $\mathbb{R}^{+} \times[0, T]$ defined on complete probability space $\left(\Omega, \mathcal{F}, \mathcal{F}_{t}, P\right)$, where $P$ is a probability measure, $\mathcal{F}$ is a $\sigma$ algebra and $\left\{\mathcal{F}_{t}\right\}_{t \geq 0}$ is a right-continuous filtration on $(\Omega, \mathcal{F})$ such that $\mathcal{F}_{0}$ contains all $P$-negligible subsets. Let $B=\left\{B(x, t) \mid x \in \mathbb{R}^{+}, t \geq 0\right\}$ be a centered Gaussian field with covariance function given by

$$
K((x, t),(y, s))=\min \{x, y\} \min \{t, s\} .
$$

We suppose that $B$ generates a $\left(\mathcal{F}_{t}, t \geq 0\right)$-martingale measure in the sense of Walsh [12]. The initial condition $q_{0}$ is supposed to be $\mathcal{F}_{0} \times \mathcal{B}\left(\mathbb{R}^{+}\right)$measurable, where $\mathcal{B}\left(\mathbb{R}^{+}\right)$is the Borelian $\sigma$-algebra over $\mathbb{R}^{+}$. Now, we mention some definitions and known results. 
Definition 1. The Caputo fractional derivative of order $\alpha \in(0,1)$ is defined by the integral operator

$$
\mathcal{D}_{t}^{\alpha} q(x, t)=\frac{1}{\Gamma(1-\alpha)} \int_{0}^{t} \frac{q_{\tau}(x, \tau)}{(t-\tau)^{\alpha}} d \tau .
$$

Definition 2. The Fourier-Laplace transform is defined as follows

$$
\widehat{q}(k, t)=\int_{0}^{\infty} e^{-i k x} q(x, t) d x, \quad \operatorname{Im}(k)<0 .
$$

The inverse Fourier-Laplace transform is

$$
q(x, t)=\frac{1}{2 \pi} \int_{-\infty}^{\infty} e^{i k x} \widehat{q}(k, t) d k .
$$

Definition 3. The two-parametric Mittag-Leffler function is defined by the power series

$$
E_{\alpha, \beta}(z)=\sum_{k=0}^{\infty} \frac{z^{k}}{\Gamma(\alpha k+\beta)}, \quad \alpha>0, \beta \in \mathbb{R} .
$$

The following theorem its going to be used to determine the domain of analyticity for the functions involved in the integral representation of the solutions to the linear problem.

Theorem 4. If $\alpha<2, \beta$ is an arbitrary real number, $\mu$ is such that $\pi \alpha / 2<\mu<\min \{\pi, \pi \alpha\}$ and $C$ is a real constant, then

$$
\begin{gathered}
\left|E_{\alpha, \beta}(z)\right| \leq \frac{C}{1+|z|}, \\
\mu \leq|\arg z| \leq \pi .
\end{gathered}
$$

The proof can be found in [10].

Now, we state an important relation between the Fourier-Laplace transform and the Mittag-Leffler function, the proof can be seen in [5].

Lemma 5. The following formula is true

$$
\frac{1}{2 \pi} \int_{-\infty}^{\infty} e^{i s t} \frac{(i s)^{\alpha-\beta}}{(i s)^{\alpha}-z} d s=t^{\beta-1} E_{\alpha, \beta}\left(z t^{\alpha}\right), \quad\left|z /(i s)^{\alpha}\right|<1,
$$

where $\operatorname{Re}(z) \leq 0, \beta>0$ and $0<\alpha<1$. 
The following lemma is going to be used by proved existence and uniqueness of the problem (2).

Lemma 6. Gronwall's lemma. Suppose $\phi_{1}, \phi_{2}, \ldots:[0, T] \rightarrow \mathbb{R}^{+}$are measurable and non-decreasing. Suppose also that there exist a constant $A$ such that for all integers $n \geq 1$, and $t \in[0, T]$,

$$
\phi_{n+1}(t) \leq A \int_{0}^{t} \phi_{n}(s) d s .
$$

Then,

$$
\phi_{n}(t) \leq \phi_{1}(t) \frac{(A t)^{n-1}}{(n-1) !} .
$$

\section{Linear Problem}

We consider the homogeneous linear problem associated with (2),

$$
\begin{cases}\mathcal{D}_{t}^{\alpha} q=a q_{x x}+(b-a) q_{x}-b q, & x>0, t \in[0, T], \\ q(x, 0)=q_{0}(x), & x>0, \\ q(0, t)=g_{0}(t), & t>0,\end{cases}
$$

where $\mathcal{D}_{t}^{\alpha}$ is the Caputo fractional derivative defined by (3).

Applying the Fourier-Laplace transform with respect to $x$ in the equation (5), we obtain

$$
\mathcal{D}_{t}^{\alpha} \hat{q}(k, t)=p(k) \hat{q}(k, t)-a q_{x}(0, t)+(a-b-i a k) g_{0}(t),
$$

where $p(k)=-a k^{2}+i(b-a) k-b$. Now, we apply the Fourier-Laplace transform to the above equation with respect to $t$ to get

$$
\hat{\hat{q}}(k, s)=\frac{1}{(i s)^{\alpha}-p(k)}\left((i s)^{\alpha-1} \hat{q}_{0}(k)-a \hat{q}_{x}(0, s)+(a-b-i a k) \tilde{g}_{0}(s)\right) .
$$

Then, using the inverse Fourier-Laplace transform in above equation with respect to $t$ and Lemma 5 , we arrive to the equation

$$
\hat{q}(k, t)=E_{\alpha, 1}\left(p(k) t^{\alpha}\right) \hat{q}_{0}(k)-a \tilde{g}_{1}(p(k), t)+(a-b-i a k) \tilde{g}_{0}(p(k), t),
$$

where $E_{\alpha, \beta}(z)$ is defined in (4), and

$$
\tilde{g}_{j}(p(k), t)=\int_{0}^{t}(t-\xi)^{\alpha-1} E_{\alpha, \alpha}\left(p(k)(t-\xi)^{\alpha}\right) \partial_{x}^{j} q(0, \xi) d \xi, \quad \text { for } j=0,1 .
$$


Finally, applying the inverse Fourier-Laplace transform in (6) with respect to $x$, we arrive to the integral representation of the solution

$$
\begin{aligned}
q(x, t)= & \frac{1}{2 \pi} \int_{-\infty}^{\infty} e^{i k x} E_{\alpha, 1}\left(p(k) t^{\alpha}\right) \hat{q}_{0}(k) d k \\
& -\frac{a}{2 \pi} \int_{-\infty}^{\infty} e^{i k x} \tilde{g}_{1}(p(k), t) d k \\
& +\frac{1}{2 \pi} \int_{-\infty}^{\infty} e^{i k x}(a-b-i a k) \tilde{g}_{0}(p(k), t) d k .
\end{aligned}
$$

Now, we consider the following region

$$
D=\left\{k=k_{R}+i k_{I} \in \mathbb{C}:\left(k_{I}+\frac{b-a}{2 a}\right)^{2}-k_{R}^{2}<\left(\frac{b+a}{2 a}\right)^{2}\right\} .
$$

Let's notice that if $k \in D$, then $\operatorname{Re}(p(k))<0$. Then, in virtue of the Theorem 4 ,

$$
\left|E_{\alpha, \beta}\left(p(k) t^{\alpha}\right)\right| \rightarrow 0, \quad \text { if }|k| \rightarrow \infty, \quad \text { for } k \in D \text { and } t>0,
$$

thus we assure the absolute convergence of the integrals above in the region $D$. Therefore, using the Cauchy theorem we can deform the contour of integration to $\partial D$ in the equation (7),

$$
\begin{aligned}
q(x, t) & =\frac{1}{2 \pi} \int_{-\infty}^{\infty} e^{i k x} E_{\alpha, 1}\left(p(k) t^{\alpha}\right) \hat{q}_{0}(k) d k \\
& -\frac{a}{2 \pi} \int_{\partial D} e^{i k x} \widetilde{g}_{1}(p(k), t) d k \\
& +\frac{1}{2 \pi} \int_{\partial D} e^{i k x}(a-b-i a k) \widetilde{g}_{0}(p(k), t) d k .
\end{aligned}
$$

Making the change of variable $k \rightarrow k^{*}=-k-i \frac{b-a}{a}$ in (6) and using that $p(k)$ is invariant under this change, we obtain

$$
a \widetilde{g}_{1}(p(k), t)=E_{\alpha, 1}\left(p(k) t^{\alpha}\right) \hat{q}_{0}\left(k^{*}\right)+(2 a-2 b+i a k) \widetilde{g}_{0}(p(k), t)-\hat{q}\left(k^{*}, t\right) .
$$

Moreover, by the Cauchy theorem

$$
\frac{1}{2 \pi} \int_{\partial D} e^{i k x} \hat{q}\left(k^{*}, t\right) d k=0 .
$$

Then, we substitute $\widetilde{g}_{1}(p(k), t)$ from equation (9) in equation (8) and using the above equation we arrive to 


$$
\begin{aligned}
q(x, t)= & \frac{1}{2 \pi} \int_{-\infty}^{\infty} e^{i k x} E_{\alpha, 1}\left(p(k) t^{\alpha}\right) \hat{q}_{0}(k) d k \\
& -\frac{1}{2 \pi} \int_{\partial D} e^{i k x} E_{\alpha, 1}\left(p(k) t^{\alpha}\right) \hat{q}_{0}\left(k^{*}\right) d k \\
& +\frac{1}{2 \pi} \int_{\partial D} e^{i k x}(b-a-i 2 a k) \widetilde{g}_{0}(p(k), t) d k .
\end{aligned}
$$

Therefore, by Fubini's theorem, we find the following integral representation for the solution

$$
q(x, t)=\int_{0}^{\infty} G^{I}(x, y, t) q_{0}(y) d y+\int_{0}^{t} G^{B}(x, t-\xi) g_{0}(\xi) d \xi
$$

where the Green function is given by

$$
\begin{aligned}
G^{I}(x, y, t)= & \frac{1}{2 \pi} \int_{-\infty}^{\infty} e^{i k(x-y)} E_{\alpha, 1}\left(p(k) t^{\alpha}\right) d k \\
& -\frac{1}{2 \pi} \int_{\partial D} e^{i k(x+y)-\frac{\mathrm{b}-\mathrm{a}}{\mathrm{a}} y} E_{\alpha, 1}\left(p(k) t^{\alpha}\right) d k
\end{aligned}
$$

and

$$
G^{B}(x, t-\xi)=\frac{1}{2 \pi} \int_{\partial D}(b-a-i 2 a k) e^{i k x}(t-\xi)^{\alpha-1} E_{\alpha, \alpha}\left(p(k)(t-\xi)^{\alpha}\right) d k .
$$

\section{Main Problem}

In this section, we prove the local existence and uniqueness of solutions for the stochastic initial-boundary value problem

$$
\left\{\begin{array}{l}
\mathcal{D}_{t}^{\alpha} q(x, t)=a q_{x x}+(b-a) q_{x}-b q+\mathcal{N} q+\dot{B}, x>0, t \in[0, T] \\
q(x, 0)=q_{0}(x) \\
q(0, t)=g_{0}(t)
\end{array}\right.
$$

where $\mathcal{N}$ is a lipschitzian operator and $\dot{B}(x, t)$ is the white noise. 
We understand the equation (10) in the Walsh [12] sense. That is, $q$ is called a solution if for all $x \in \mathbb{R}^{+}$and $t \in[0, T], q$ satisfies

$$
\begin{aligned}
q(x, t)= & \int_{0}^{\infty} G^{I}(x, y, t) q_{0}(y) d y+\int_{0}^{t} G^{B}(x, t-\xi) g_{0}(\xi) d \xi \\
& +\int_{0}^{t} \int_{0}^{\infty} G(x-y, t-\xi) \mathcal{N} q(y, \xi) d y d \xi \\
& +\int_{0}^{t} \int_{0}^{\infty} G(x-y, t-\xi) d B(y, \xi),
\end{aligned}
$$

where

$$
G(x, t)=\frac{1}{2 \pi} \int_{-\infty}^{\infty} e^{i k x} E_{\alpha, 1}\left(p(k) t^{\alpha}\right) d k .
$$

The field $\left\{q(x, t) \mid x \in \mathbb{R}^{+}, t \geq 0\right\}$ is said to be a global mild solution of equation (11) if, for all $0<T<\infty,\left\{q(x, t) \mid x \in \mathbb{R}^{+}, t \in[0, T]\right\}$ is a mild solution on the interval $[0, T]$. Furthermore, a global mild solution is in $L^{p}(\Omega)$ for some $p \geq 1$ if, for all $T \geq 0, x \in \mathbb{R}^{+}$, and for all $t \in[0, T], \sup \left\{\mathbb{E}\left(|q(x, t)|^{p}\right) \mid(x, t) \in\right.$ $\left.\mathbb{R}^{+} \times[0, T]\right\}<\infty$, where $\mathbb{E}$ is the expectation with respect to $P$.

We are going to prove the existence and uniqueness theorem, where we understand the uniqueness of the solutions in (11) in the sense that for any mild solutions $q_{1}$ and $q_{2}$ on $[0, T]$ we have $q_{1}(x, t)=q_{2}(x, t)$ in $L^{p}(\Omega), p \geq 1$, for all $x \in \mathbb{R}^{+}$and for all $t \in[0, T]$.

Theorem 7. Suppose that for each $T>0$, there exists a constant $C>0$ such that for each $(x, t) \in \mathbb{R}^{+} \times[0, T]$,

$$
\left|\mathcal{N} q_{1}-\mathcal{N} q_{2}\right| \leq C\left|q_{1}-q_{2}\right|,
$$

and for some $p \geq 1$,

$$
\sup _{x \in \mathbb{R}^{+}} \mathbb{E}\left(\left|q_{0}(x)\right|^{p}\right)<\infty .
$$

Then, there exists a unique solution $q(x, t)$ for the problem (10). Moreover, for all $T>0$ and $p \geq 1$,

$$
\sup _{(x, t) \in \mathbb{R}^{+} \times[0, T]} \mathbb{E}\left(|q(x, t)|^{p}\right)<\infty .
$$

Proof. First, we are going to prove that $\left\{q^{n}(x, t)\right\}_{n \geq 0}$ is a Cauchy sequence 
in the complete space $L^{p}(\Omega)$, where

$$
\begin{aligned}
q^{n+1}(x, t)= & q^{0}(x, t)+\int_{0}^{t} G^{B}(x, t-\xi) g_{0}(\xi) d \xi \\
& +\int_{0}^{t} \int_{0}^{\infty} G(x-y, t-\xi) \mathcal{N} q^{n}(y, \xi) d y d \xi \\
& +\int_{0}^{t} \int_{0}^{\infty} G(x-y, t-\xi) d B(y, \xi) .
\end{aligned}
$$

Here, the Green function $G$ is given by

$$
G(x, t)=\frac{1}{2 \pi} \int_{-\infty}^{\infty} e^{i k x} E_{\alpha, 1}\left(p(k) t^{\alpha}\right) d k
$$

and

$$
q^{0}(x, t)=\int_{0}^{\infty} G^{I}(x, y, t) q_{0}(y) d y .
$$

We have for $n \geq 2$,

$$
\begin{aligned}
& \mathbb{E}\left(\left|q^{n+1}(x, t)-q^{n}(x, t)\right|^{p}\right) \\
& =\mathbb{E}\left(\left|\int_{0}^{t} \int_{0}^{\infty} G(x-y, t-\xi)\left[\mathcal{N} q^{n}(y, \xi)-\mathcal{N} q^{n-1}(y, \xi)\right] d y d \xi\right|^{p}\right) \\
& \leq C(p) \int_{0}^{t} \int_{0}^{\infty} G(x-y, t-\xi) \mathbb{E}\left(\left|q^{n}(y, \xi)-q^{n-1}(y, \xi)\right|^{p}\right) d y d \xi \\
& \leq C(p) \int_{0}^{t} \sup _{y \in \mathbb{R}^{+}} \mathbb{E}\left(\left|q^{n}(y, \xi)-q^{n-1}(y, \xi)\right|^{p}\right) d \xi
\end{aligned}
$$

and by Minkowski inequality and (12),

$$
\begin{aligned}
& \sup _{x \in \mathbb{R}^{+}} \mathbb{E}\left(\left|q^{1}(x, t)-q^{0}(x, t)\right|^{p}\right) \\
\leq & C(p)\left(\sup _{x \in \mathbb{R}^{+}} \mathbb{E}\left(\left|q^{1}(x, t)\right|^{p}\right)+\sup _{x \in \mathbb{R}^{+}} \mathbb{E}\left(\left|q^{0}(x, t)\right|^{p}\right)\right)<\infty .
\end{aligned}
$$

Then, Gronwall lemma shows that

$$
\sum_{n \geq 0} \sup _{(x, t) \in \mathbb{R}^{+} \times[0, T]} \mathbb{E}\left(\left|q^{n}(x, t)-q^{n-1}(x, t)\right|^{p}\right)<\infty .
$$


Hence, $\left\{q^{n}(x, t)\right\}_{n \geq 0}$ is a Cauchy sequence in $L^{p}(\Omega)$. Let

$$
q(x, t)=\lim _{n \rightarrow \infty} q^{n}(x, t) .
$$

Then for each $(x, t) \in \mathbb{R}^{+} \times[0, T]$,

$$
\sup _{(x, t) \in \mathbb{R}^{+} \times[0, T]} \mathbb{E}\left(|q(x, t)|^{p}\right)<\infty .
$$

Take $n \rightarrow \infty$ in $L^{p}(\Omega)$ at both sides of (13). Then, it shows that $q(x, t)$, satisfies the problem (10). Finally, we prove the uniqueness of the solution. Let $q_{1}$ and $q_{2}$ be the two solutions of problem (10), then

$$
\begin{gathered}
\mathbb{E}\left(\left|q_{1}(x, t)-q_{2}(x, t)\right|^{p}\right) \\
=\mathbb{E}\left(\left|\int_{0}^{t} \int_{0}^{\infty} G(x-y, t-\xi)\left[\mathcal{N} q_{1}(y, \xi)-\mathcal{N} q_{2}(y, \xi)\right] d y d \xi\right|^{p}\right) \\
\leq C(p) \int_{0}^{t} \int_{0}^{\infty} G(x-y, t-\xi) \mathbb{E}\left(\left|q_{1}(y, \xi)-q_{2}(y, \xi)\right|^{p}\right) d y d \xi \\
\leq C(p) \int_{0}^{t} \sup _{y \in \mathbb{R}^{+}} \mathbb{E}\left(\left|q_{1}(y, \xi)-q_{2}(y, \xi)\right|^{p}\right) d \xi .
\end{gathered}
$$

The Gronwall lemma yields that

$$
\mathbb{E}\left(\left|q_{1}(x, t)-q_{2}(x, t)\right|^{p}\right)=0 .
$$

The Theorem 7 is proved.

\section{References}

[1] J. Ahn, S. Kang,Y. Kwon, A Laplace transform finite difference method for the BlackScholes equation, Mathematical and Computer Modelling, 51, No. 3 (2010), 247-255.

[2] F. Black, M. Scholes, The pricing of options and corporate liabilities, The journal of political economy, 81, (1973), 637-654.

[3] W. Chen, X. Xu, S.P. Zhu, Analytically pricing double barrier options based on a timefractional Black-Scholes equation. Computers and Mathematics with Applications, 69, No. 12 (2015), 1407-1419.

[4] A. Fokas, A Unified Approach to Boundary Value Problems, SIAM, USA (2008).

[5] R. Gorenflo, A. A. Kilbas, F. Mainardi, S. V. Rogosin, Mittag-Leffler Functions, Related Topics and Applications, Springer-Verlag, Germany (2014). 
[6] L. Jódar, P. Sevilla-Peris, J. C. Cortés, R. Sala, A new direct method for solving the Black-Scholes equation, Applied mathematics letters, 18, No. 1 (2005), 29-32.

[7] S. Kumar, A. Yildirim, Y. Khan, H. Jafari, K. Sayevand, L. Wei, Analytical solution of fractional Black-Scholes European option pricing equation by using Laplace transform, Journal of fractional calculus and Applications, 2, No. 8 (2012), 1-9.

[8] H. K. Liu, J. J. Chang, A closed-form approximation for the fractional Black-Scholes model with transaction costs, Computers and Mathematics with Applications, 65, No. 11 (2013), 1719-1726.

[9] B. O. Osu, A. I. Chukwunezu, On the solution to a fractional Black-Scholes equation for the price of an option, International Journal of Mathematical Analysis and Applications, 1, No. 3 (2014), 38-42.

[10] I. Podlubny, Fractional Differential Equations, Academic Press, San Diego, USA (1999).

[11] L. Song, W. Wang, Solution of the fractional Black-Scholes option pricing model by finite difference method, Abstract and Applied Analysis, 2013, (2013), 1-10.

[12] J. B. Walsh, An introduction to stochastic partial differential equations, Lecture Notes in Math, 1180, (1986), 265-439.

[13] H. Windcliff, P. A. Forsyth, K. R. Vetzal, Analysis of the stability of the linear boundary condition for the Black-Scholes equation, Journal of Computational Finance, 8, (2004), 65-92.

[14] C. Zeng, Y. Chen, Q. Yang, Almost sure and moment stability properties of fractional order Black-Scholes model, Fractional Calculus and Applied Analysis, 16, No. 2 (2013), 317-331. 